\title{
Identification of soluble thrombomodulin and tissue plasminogen activator-inhibitor complex as biomarkers for prognosis and early evaluation of septic shock and sepsis-induced disseminated intravascular coagulation
}

\author{
Jin Zhang ${ }^{1 \#}$, Mingming Xue ${ }^{1 \#}$, Yao Chen ${ }^{1 \#}$, Chenglong Liu ${ }^{2 \#}$, Zhongshu Kuang ${ }^{1}$, Sucheng Mu ${ }^{1}$, Wei Wei ${ }^{1}$, \\ Jun Yin ${ }^{1}$, Hao Xiang ${ }^{1}$, Yanyan Hu ${ }^{1}$, Xiangyu Long ${ }^{1}$, Shuo Fang ${ }^{1}$, Si Sun ${ }^{1}$, Beili Wang ${ }^{3}$, Chaoyang Tong ${ }^{1}$, \\ Zhenju Song ${ }^{1}$
}

${ }^{1}$ Department of Emergency Medicine, Zhongshan Hospital, Fudan University, Shanghai, China; ${ }^{2}$ Rizhao Hospital of Traditional Chinese Medicine, Rizhao, China; ${ }^{3}$ Department of Laboratory Medicine, Zhongshan Hospital, Fudan University, Shanghai, China

Contributions: (I) Conception and design: Z Song, C Tong, B Wang; (II) Administrative support: Z Song, C Tong, B Wang; (III) Provision of study materials or patients: J Zhang, M Xue, Z Kuang, S Mu, W Wei, J Yin, H Xiang, Y Hu, X Long, S Fang, S Sun; (IV) Collection and assembly of data: C Liu, B Wang; (V) Data analysis and interpretation: M Xue, J Zhang, Y Chen; (VI) Manuscript writing: All authors; (VII) Final approval of manuscript: All authors.

\#These authors contributed equally to this work.

Correspondence to: Zhenju Song. Department of Emergency Medicine, Zhongshan Hospital, Fudan University, Shanghai, China. Email: song.zhenju@zs-hospital.sh.cn; Chaoyang Tong. Department of Emergency Medicine, Zhongshan Hospital, Fudan University, Shanghai, China. Email: tong.chaoyang@zs-hospital.sh.cn; Beili Wang. Department of Laboratory Medicine, Zhongshan Hospital, Fudan University, Shanghai, China. Email: wang.beili@zs-hospital.sh.cn.

Background Endothelium injury and coagulation dysfunction play an important role in the pathogenesis of sepsis. Soluble thrombomodulin (sTM), tissue plasminogen activator-inhibitor complex (t-PAIC), thrombin-antithrombin complex (TAT) and $\alpha 2$-plasmin inhibitor-plasmin complex (PIC) are biomarkers of endothelium injury and coagulation dysfunction. This study aimed to explore the prognostic values and diagnostic performance for septic shock and sepsis-induced disseminated intravascular coagulation (DIC) of endothelial biomarkers.

Methods: We conducted an observational study on patients with sepsis admitted to intensive care unit (ICU) at a teaching hospital from January 2016 to December 2018. Levels of sTM, t-PAIC, TAT and PIC were measured at admission day and day 5-7 after admission and detected by qualitative chemiluminescence enzyme immunoassay performed on HISCL automated analyzers.

Results: A total of 179 septic patients and 125 non-septic ICU controls were enrolled. The level of sTM was higher in septic patients compared to ICU controls (OR =1.093, 95\% CI: 1.045-1.151, $\mathrm{P}<0.001$ ). Moreover, higher levels of sTM and t-PAIC were independent predictors of poor 60-day prognosis for septic patients ( $\mathrm{HR}=1.012$, 95\% CI: 1.003-1.022, $\mathrm{P}=0.012$; $\mathrm{HR}=1.014, \mathrm{P}=0.009$ ). Level of sTM was also higher in patients with septic shock as revealed by multivariate analysis (OR $=1.049,95 \%$ CI: 1.020-1.078, $\mathrm{P}=0.001)$, as well as in patients with sepsis-induced DIC (OR $=1.109,95 \%$ CI: $1.065-1.158, \mathrm{P}<0.001)$. sTM was considered as a sensitive biomarker for the early prediction of septic shock and sepsis-induced DIC, with AUC up to 0.765 (0.687-0.842) and 0.864 (0.794-0.935) of receiver operating characteristic curve.

Conclusions: Most patients developed coagulopathy which was closely linked to endothelial injury in initial phase of sepsis, which was demonstrated by abnormalities in endothelial biomarkers and their strong association with poor 60-day prognosis and development of septic shock and sepsis-induced DIC.

Keywords: Soluble thrombomodulin (sTM); tissue plasminogen activator-inhibitor complex (t-PAIC); endothelial dysfunction; sepsis; septic shock; sepsis-induced disseminated intravascular coagulation (DIC) 
Submitted Aug 11, 2021. Accepted for publication Aug 28, 2021.

doi: 10.21037/apm-21-2222

View this article at: https://dx.doi.org/10.21037/apm-21-2222

\section{Introduction}

Sepsis is a syndrome of physiological, pathological, and biochemical abnormalities induced by infection which has become a major public health concern (1). Septic shock is defined as a subset of sepsis in which underlying circulatory and cellular metabolic abnormalities are profound enough to influence prognosis. Sepsis and septic shock have an increasing incidence and are considered as leading causes of mortality $(2,3)$, likely reflecting aging populations with more comorbidities and increased recognition due to advanced techniques and awareness of health providers (4-8). The pathogenesis of sepsis is not precisely understood, and mounting evidence suggests that an exaggerated and uncontrolled systemic host inflammatory and coagulation response to infectious pathogens leads to inflammatory injury, microvascular thrombosis, multiple organ dysfunction syndrome (MODS) and even death.

Proinflammatory cytokines secretion initiated by the host response stimulates the expression of tissue factors and activated coagulation system, which in turn modulates inflammatory activity through specific receptors such as protease-activated receptors. Considering the excessive crosstalk between inflammation and coagulation is ongoing from the onset of sepsis, severe coagulopathy may develop in early phase of sepsis, which involves dysfunction of coagulation cascade process, anticoagulant and fibrinolytic systems, as well as relevant endothelial injury and abnormality of blood cells and vascular wall function (9-11). The severity of sepsis-associated coagulopathy is variable, ranging from subclinical abnormalities that are detectable by a mild decrease in platelet count and prolongation of clotting times to severe forms of coagulopathy such as disseminated intravascular coagulation (DIC) $(10,12)$.

The function of endothelium in the pathogenesis of sepsis is regulated by pathways that control vascular permeability, coagulation, and systemic inflammation (13-15). Severe sepsis causes the upregulation of several proinflammatory adhesion molecules and the release of proinflammatory mediators, including cytokines and lipid products, and is accompanied by the dysfunction of coagulation factors, all of which disrupt the barrier of endothelial cells $(15,16)$. Although acknowledged markers such as PT, APTT, D-dimers and platelets have been widely used to identify the development of DIC, those markers have low sensitivity in early diagnosis. Endothelium injury and an abnormal coagulation system play an important role in the development of septic shock and sepsisinduced DIC. Thus, markers that reflect endothelial injury could potentially predict the early stage of septic shock and DIC (17-19). Soluble thrombomodulin (sTM), tissue plasminogen activator-inhibitor complex (t-PAIC), thrombin-antithrombin complex (TAT), and $\alpha 2$-plasmin inhibitor-plasmin complex (PIC) are considered to be sensitive markers of endothelial cell injury and coagulation disorders. Recent studies have found that sTM, t-PAIC, TAT and PIC are related to acute kidney injury, acute respiratory distress syndrome (ARDS) and DIC (20-22). However, it is not clear whether these markers can predict the early occurrence of septic shock or sepsis-related DIC. Therefore, this study intended to investigate these biomarkers as predictors for 60 -day mortality in patients with sepsis and evaluate the predictive value of endothelial markers for early prediction of septic shock and sepsisinduced DIC.

We present the following article in accordance with the STROBE reporting checklist (available at https://dx.doi. org/10.21037/apm-21-2222).

\section{Methods}

\section{Patients and study design}

A retrospective study was carried out in the emergency ICU of Zhongshan Hospital, Fudan University, Shanghai, China. Patients diagnosed with sepsis on admission from January 2016 to December 2018 were enrolled in this study. The diagnosis of sepsis referred to The Third International Consensus Definitions for Sepsis and Septic Shock (Sepsis-3), namely suspected infection with Sequential Organ Failure Assessment (SOFA) score $\geq 2$ (23). DIC was diagnosed according to JAAM criteria (score $\geq 4$ ) (24). All patients were screened for prognosis at day 60 after admission and development of shock and DIC. Septic patients were classified into septic shock and nonseptic shock groups, DIC and non-DIC groups according 
to developing septic shock or DIC during the first 7 days of ICU stay or not.

Non-septic patients in critical condition admitted to the emergency ICU were recruited as ICU controls, whose diagnoses included (I) acute heart failure ( $\mathrm{n}=29)$, (II) asthma ( $\mathrm{n}=19)$, (III) acute exacerbation of chronic obstructive pulmonary disease $(\mathrm{n}=18)$, (IV) pneumothorax $(\mathrm{n}=13),(\mathrm{V})$ hypertensive emergencies $(\mathrm{n}=16),(\mathrm{VI})$ arrhythmia $(\mathrm{n}=16)$, (VII) acute stroke ( $\mathrm{n}=8)$ and (VIII) intoxication $(\mathrm{n}=6)$, which were not associated with acute coagulation dysfunction and endothelium injury. The exclusion criteria for both septic patients and ICU controls were as follows: (I) age $<18$ years; (II) immune system disease; (III) malignancy; (IV) hematological disease; (V) thrombotic disease; (VI) chronic liver disease (Child-Pugh C); (VII) chronic renal failure requiring hemodialysis. The study was conducted in accordance with the Declaration of Helsinki (as revised in 2013). The study was approved by the Institutional Research Ethics Committee of Zhongshan Hospital, Fudan University (No: 2006-23). Informed consent for the use of biological samples were acquired from patients or their authorized agents on admission as this study is part of an on-going project regarding pathogenesis of sepsis since 2006 , in spite of the retrospective nature of this study. Sepsis treatment was according to Surviving Sepsis Campaign: International Guidelines for Management of Sepsis and Septic Shock: 2016 (25).

\section{Data collection}

Baseline characteristics including age, gender, site of infection, acute physiology and chronic health evaluation II (APACHE II) and sequential organ failure assessment (SOFA) scores, requirement for mechanical ventilation and the 60-day prognosis of patients were collected from the Electronic Medical Record System (EMRS) and our sepsis database, which we founded in 2006 to routinely collect the sample and information of patients with sepsis. Underlying medical history was also obtained including ischemic heart disease, chronic heart failure, chronic obstructive pulmonary disease, cerebrovascular accident and diabetes mellitus. The levels of prothrombin time (PT), thrombin time (TT), activated partial thromboplastin time (APTT), fibrinogen (FIB), D-dimer, platelet count (PLT) and lactate were collected. Indexes of blood coagulation including PT, TT, APTT and FIB were assayed using the CS-2100i automatic coagulation analyzer (Sysmex, Japan).

\section{Biomarker measurement}

2-3 mL venous blood was drawn from patients and preserved in a sample tube (BD Vacutainer with sodium citrate anticoagulant, UK). Processing of samples was carried out according to standardized protocol for the measurement of coagulation indexes of central laboratory of Zhongshan Hospital, Fudan University, which has been approved by ISO15189. The samples were centrifuged at $1,500 \mathrm{~g}$ at $4^{\circ} \mathrm{C}$ for $10 \mathrm{~min}$, and then $500 \mu \mathrm{L}$ supernatant was collected and stored at $-80^{\circ} \mathrm{C}$. The frozen plasma samples were thawed just before use, by immersion in a water bath at $37^{\circ} \mathrm{C}$ for $5 \mathrm{~min}$. The levels of sTM, t-PAIC, TAT and PIC were measured via qualitative chemiluminescence enzyme immunoassay performed on HISCL automated analyzers (Sysmex, Japan), which has been commercialized and approved by Pharmaceuticals and medical devices agency of Japan, National Medical Products Administration of China and acquired CE permission in European Union to be used for aided diagnosis and disease monitoring. The examination methods were detailed in the product instructions (HISCL sTM, t-PAIC, TAT and PIC Assay Kit, Japan).

\section{Statistical analysis}

Normally distributed continuous variables were expressed as the means \pm standard deviations, and non-normally distributed continuous variables were expressed as medians (25th and 75th quartiles). Student's t test or oneway analysis of variance was used to compare normally distributed continuous variables. Kruskal-Wallis oneway analysis or Mann-Whitney $U$ test was utilized to compare nonnormally distributed continuous variables. Categorical data were expressed as numbers (percentages) and compared by Pearson's chi-square test or Fisher's exact test when appropriate. Multivariate logistic regression model based on a forward stepwise method was used to identify the independent factors. Cox proportional hazards regression analyses based on a forward stepwise method was used to estimate the hazard ratios with $95 \%$ CIs to discover prognostic markers. Receiver operating characteristic (ROC) curves were constructed and the areas under the ROC curves (AUCs) were determined. The optimal cutoff values with highest Youden index were calculated to maximize the sum of the sensitivity and specificity. KaplanMeier estimates were used to illustrate trends in 60-day 


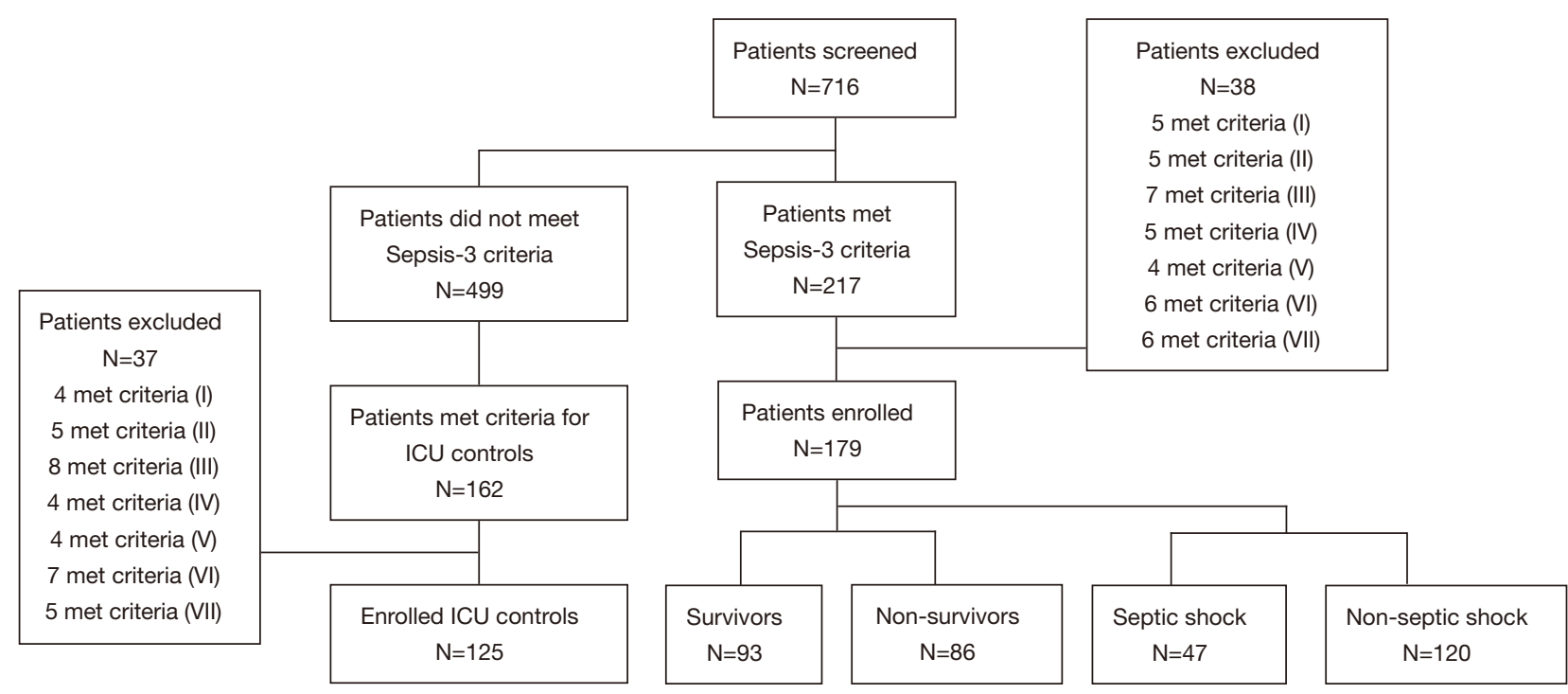

Figure 1 Flow chart of included patients and ICU controls.

mortality, and Log-rank tests were performed to compare survival curves. All statistical analyses were two-tailed, and the significance level was set to $\mathrm{P}<0.05$. Power analysis was conducted on http://powerandsamplesize.com based on a significance level $\alpha=0.05$ and results have been shown in Table S1. Statistical analyses were performed using SPSS (version 22.0, SPSS, Chicago, IL, USA).

\section{Results}

\section{Levels of endotbelial injury and hemostatic biomarkers in septic patients and ICU controls}

A total of 179 septic patients and 125 non-septic ICU controls were enrolled (Figure 1). The baseline characteristics of study population were shown in Table 1. Septic patients had longer ICU and hospital stays and higher mortality rates, APACHE II and SOFA scores. For the hemostatic parameters, the levels of PLT, PT, TT, APTT, FIB, and D-dimer all showed statistical difference between septic patients and controls. Moreover, sTM (19.9 vs. $11.1 \mathrm{TU} / \mathrm{mL}, \mathrm{P}<0.001)$, t-PAIC (18.1 vs. $7.2 \mathrm{ng} / \mathrm{mL}, \mathrm{P}<0.001)$ and TAT $(19.4$ vs. $8.7 \mathrm{ng} / \mathrm{mL}$, $\mathrm{P}<0.0001)$ levels were higher in septic patients. Then, we performed multivariate analysis and found that difference in levels of APACHE II score (OR =1.112, 95\% CI: 1.051 1.176, $\left.\mathrm{P}_{\text {adj }}<0.001\right)$, D-dimer (OR $=1.097,95 \%$ CI: 1.045 $\left.1.151, \mathrm{P}_{\mathrm{adj}}<0.001\right)$ and sTM (OR $=1.093,95 \%$ CI: 1.049 $\left.1.139, \mathrm{P}_{\mathrm{adj}}<0.001\right)$ between two groups remained statistically significant. These results suggested the endothelial cell injury and coagulation dysfunction during the acute early phase of sepsis.

\section{sTM and t-PAIC as predictors for 60-day mortality in patients with sepsis}

According to 60-day mortality, septic patients were divided into survival $(\mathrm{n}=93)$ and non-survival groups $(\mathrm{n}=86)$. In univariate analyses, age, APACHE II and SOFA scores, the levels of PT, TT, D-dimer, sTM, TAT, t-PAIC and lactate of non-survivors were higher than those of survivors (Table 2). As revealed by Cox regression analysis, APACHE II score (HR $=1.052,95 \%$ CI: 1.029-1.075, $\left.\mathrm{P}_{\text {adj }}<0.001\right)$, age (HR $=1.027,95 \%$ CI: 1.013-1.041, $\left.\mathrm{P}_{\text {adj }}<0.001\right)$, sTM (HR $=1.012,95 \%$ CI: $\left.1.003-1.022, \mathrm{P}_{\mathrm{adj}}=0.012\right)$ and t-PAIC (HR $=1.014,95 \%$ CI: $\left.1.004-1.025, \mathrm{P}_{\text {adj }}=0.009\right)$ were independent predictors for 60-day mortality of septic patients.

Analyzing the performance of age, sTM, t-PAIC and APACHE II score for predicting 60-day mortality, the values of the AUC were 0.681 (0.602-0.760), 0.697 (0.620-0.775), $0.716(0.642-0.790)$ and $0.747(0.675-$ 0.818), respectively. Combining the APACHE II score with age, sTM and t-PAIC, the AUC raised up to 0.809 $(0.747-0.872, \mathrm{P}<0.001)$ (Figure $2 A)$. The median values of sTM and t-PAIC of septic patients of non-survivors were 19.0 TU/mL and $18.3 \mathrm{ng} / \mathrm{mL}$, respectively. According to the median values, we plotted the Kaplan-Meier curves. Septic patients with sTM, t-PAIC concentrations higher 
Table 1 Baseline characteristic, endothelial cell injury and coagulation markers of septic and ICU-control patients

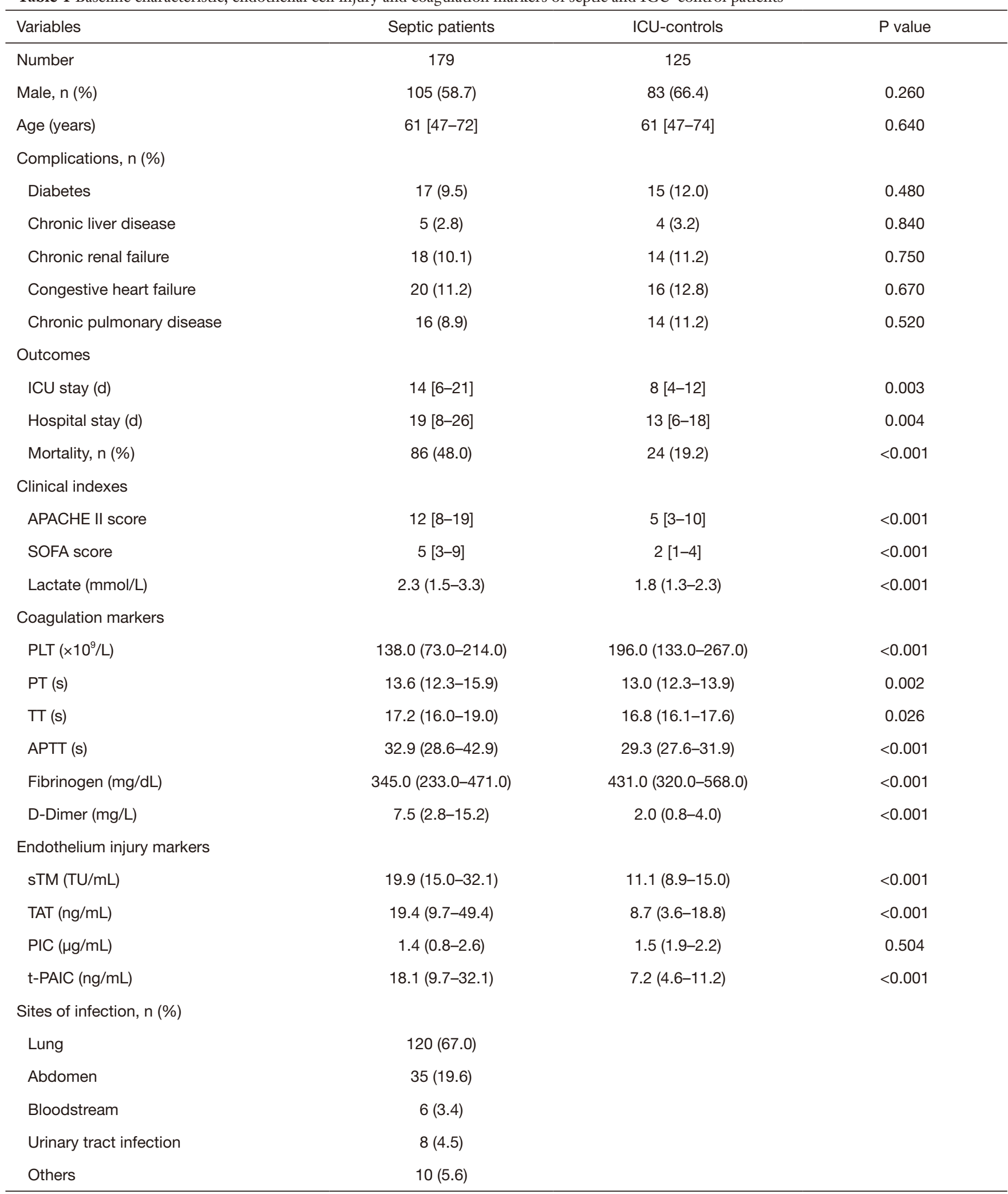

Table 1 (continued) 
Table 1 (continued)

\begin{tabular}{lc}
\hline Variables & Septic patients \\
\hline Microbiology positive, $\mathrm{n}(\%)$ & $70(39.1)$ \\
Gram-positive & $27(38.6)$ \\
Gram-negative & $31(44.3)$ \\
Fungi & $4(5.7)$ \\
Mixed & $8(11.4)$ \\
Microbiology unknown & $109(60.9)$ \\
\hline
\end{tabular}

APACHE, Acute Physiology and Chronic Health Evaluation; APTT, activated partial thromboplastin time; PIC, $\alpha 2$-plasmin inhibitor-plasmin complex; PLT, platelet; PT, prothrombin time; SOFA, Sequential Organ Failure Assessment; sTM, soluble thrombomodulin; TAT, thrombinantithrombin complex; t-PAIC, tissue plasminogen activator-inhibitor complex; TT, thrombin time.

than the median thresholds had a lower probability of 60-day survival (Figure 2B,2C).

Then, we detected dynamic changes in the biomarkers within 5-7 days after ICU admission. The SOFA score (3 vs. $4, \mathrm{P}<0.001)$, levels of sTM (14.1 vs. $18.6 \mathrm{ng} / \mathrm{mL}$, $\mathrm{P}=0.003)$, t-PAIC $(8.8$ vs. $18.2 \mathrm{ng} / \mathrm{mL}, \mathrm{P}<0.001)$ and lactate (1.5 vs. $2 \mathrm{mmol} / \mathrm{L}, \mathrm{P}=0.004)$ in survivors within $5-7$ days were significantly lower than those on the first day of ICU admission (Figure 2D). However, in the non-survival group, the SOFA score and the level of sTM and t-PAIC within 5-7 days was not found to be significantly different from those on the day of admission (Table 3).

\section{Early prediction role of sTM and lactate for septic shock}

Septic patients were divided into shock $(\mathrm{n}=47)$ and nonshock group $(\mathrm{n}=120)$. Patients diagnosed with septic shock on admission were excluded. The mortality rate, APACHE II and SOFA scores were significantly higher in patients developing septic shock (Table 4). Univariate analysis revealed that the difference in levels of PLT, PT, APTT, FIB, D-dimer, sTM, t-PAIC and lactate were statistically significant between two groups (Table 4). The levels of sTM $\left(\mathrm{OR}=1.049,95 \%\right.$ CI: $\left.1.020-1.078, \mathrm{P}_{\text {adj }}=0.001\right)$, lactate $(\mathrm{OR}$ $=1.307,95 \%$ CI: $\left.1.039-1.644, \mathrm{P}_{\mathrm{adj}}=0.022\right)$ and APACHE II score $\left(\mathrm{OR}=1.076,95 \% \mathrm{CI}: 1.019-1.137, \mathrm{P}_{\text {adj }}=0.009\right)$ remained significantly different in multivariate analysis. The evaluation of predictive value for septic shock of several markers was carried out via receiver operating curve analysis (Figure 2E). Among three markers, the AUC was 0.765 (0.687-0.842) for sTM which was higher than 0.691 (0.593$0.790)$ for lactate and $0.755(0.674-0.837)$ for APACHE II score. The AUC for the combination of markers was 0.811
$(0.738-0.884)$

\section{Early prediction role of sTM, PLT and D-dimer for sepsis- induced DIC}

Patients with sepsis-induced DIC $(\mathrm{n}=28)$ had higher severity of diseases with higher APACHE II and SOFA scores on admission ( $n=144)$. Patients diagnosed with DIC on ICU admission were excluded. The difference in levels of PLT, PT, APTT, FIB and D-dimer on admission were statistically significant between two groups. Additionally, the levels of sTM (42.05 vs. $18.4 \mathrm{TU} / \mathrm{mL}, \mathrm{P}<0.001)$ and t-PAIC (32.25 vs. $15.1 \mathrm{ng} / \mathrm{mL}, \mathrm{P}<0.001$ ) in DIC patients were significantly higher than those in non-DIC patients (Table 5). In multivariate analysis, PLT (OR $=0.970,95 \%$ CI: $0.956-$ $\left.0.984, \mathrm{P}_{\text {adj }}<0.001\right)$, sTM (OR $=1.109,95 \%$ CI: $1.063-1.158$, $\left.\mathrm{P}_{\text {adj }}<0.001\right)$ and D-dimer (OR $=1.071,95 \%$ CI: $1.015-1.129$, $\left.\mathrm{P}_{\mathrm{adj}}<0.001\right)$ are closely related to sepsis-induced DIC. Analyzing the performance of the markers for predicting DIC, the values of AUC were 0.865 (0.807-0.922), 0.864 (0.794-0.935) and 0.775 (0.695-0.856) for PLT, sTM, and D-dimer, respectively. Combining the PLT with sTM and D-dimer, the AUC increased to 0.950 (0.916-0.983) (Figure 2F).

\section{Discussion}

The current study indicated that high levels of sTM and t-PAIC were independent predictive factors for the poor outcome of septic patients. Combining the APACHE II score with age, sTM and tPAIC, the predictive value raised even higher. In addition, the levels of sTM has early predictive values of septic shock and sepsis-induced DIC. 
Table 2 Baseline characteristic and univariate analysis of the endothelial injury and coagulation markers between survival and non-survival groups

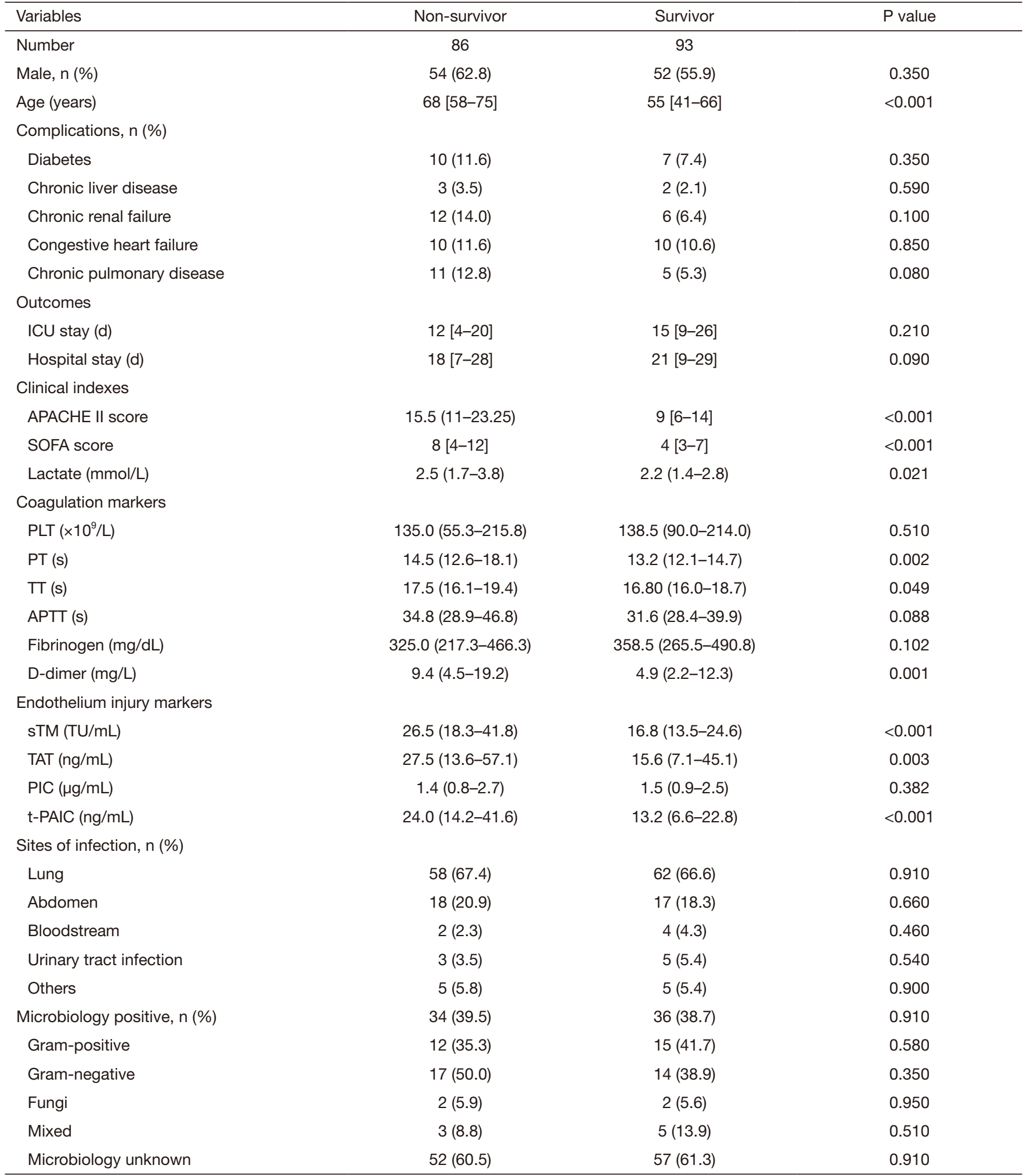

APACHE, Acute Physiology and Chronic Health Evaluation; APTT, activated partial thromboplastin time; PIC, $\alpha 2$-plasmin inhibitor-plasmin complex; PLT, platelet; PT, prothrombin time, SOFA Sequential Organ Failure Assessment; sTM, soluble thrombomodulin; TAT, thrombinantithrombin complex; t-PAIC, tissue plasminogen activator-inhibitor complex; TT, thrombin time. 
A

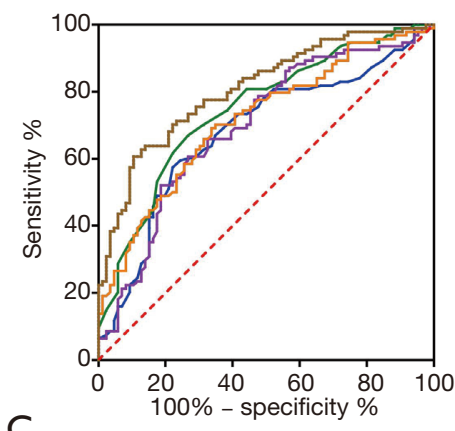

C

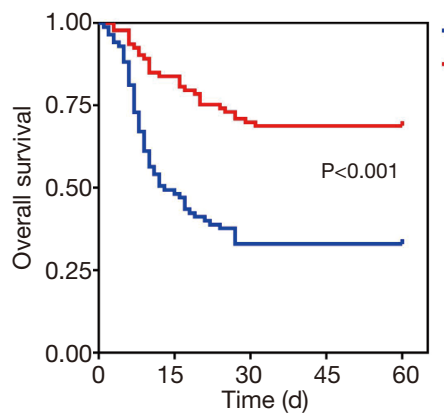

E

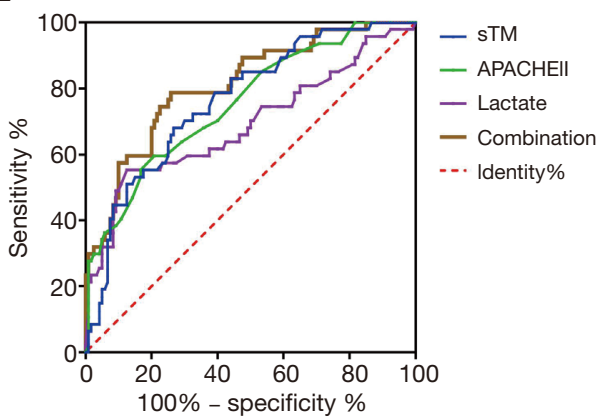

B

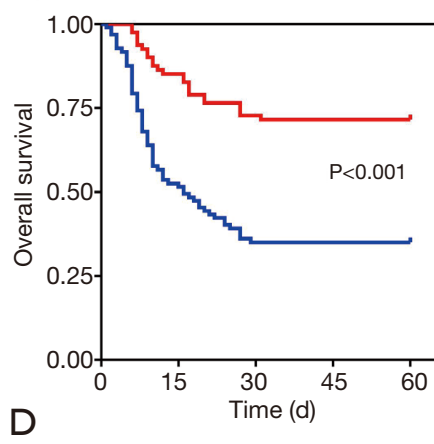

- $\mathrm{sTM}<19.0 \mathrm{TU} / \mathrm{mL}$

$-\mathrm{sTM} \geq 19.0 \mathrm{TU} / \mathrm{mL}$

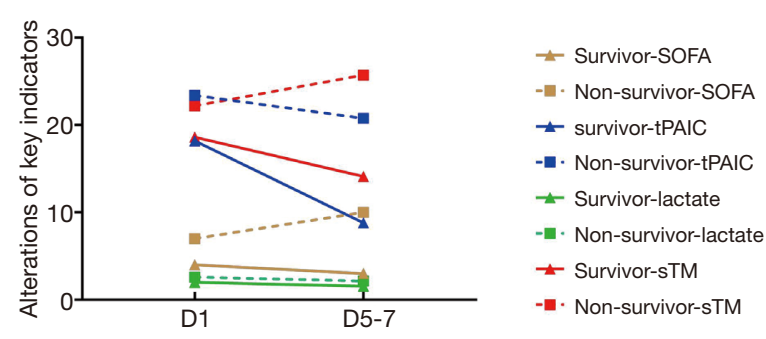

$\mathrm{F}$

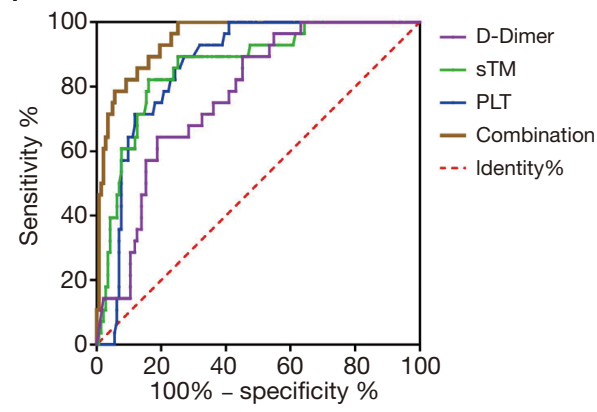

Figure 2 Soluble thrombomodulin, t-PAIC and hemostatic parameters as biomarkers for the prediction of prognosis and early evaluation of septic shock and sepsis-induced DIC. (A) ROC curves for the prediction of 60-day mortality; (B) comparison of Kaplan-Meier survival curves between patients with high and low sTM levels; (C) comparison of Kaplan-Meier survival curves between patients with high and low t-PAIC levels; (D) dynamic changes of SOFA score, levels of sTM (TU/mL), t-PAIC $(\mathrm{ng} / \mathrm{mL})$ and lactate $(\mathrm{mmol} / \mathrm{L})$ in survivors and nonsurvivors; (E) ROC curves for the prediction of septic shock; (F) ROC curves for the prediction of sepsis-induced DIC. APACHE, Acute Physiology and Chronic Health Evaluation; APTT, activated partial thromboplastin time; PLT, platelet, PT, prothrombin time; SOFA, Sequential Organ Failure Assessment; sTM, soluble thrombomodulin; t-PAIC, tissue plasminogen activator-inhibitor complex; DIC, disseminated intravascular coagulation.

These findings emphasized the importance of endothelial injury among patients with sepsis, especially in the early recognition and management of septic shock and sepsisinduced DIC.

Inflammation and coagulation constitute two host defense systems with complementary roles against infection, which means that an overwhelming systemic inflammatory reaction in sepsis is accompanied by coagulopathy, and both may contribute to tissue damage in the early phase of sepsis (26-37). In a mouse model of endotoxemia, the administration of LPS resulted in a reduction in total tissue TM antigen in the lung and brain (35). Under in vitro conditions, the addition of LPS and/or cytokines to endothelial cells has been shown to decrease the synthesis of TM, tissue-type plasminogen activator and heparan, increase the expression of tissue factor (TF) and 


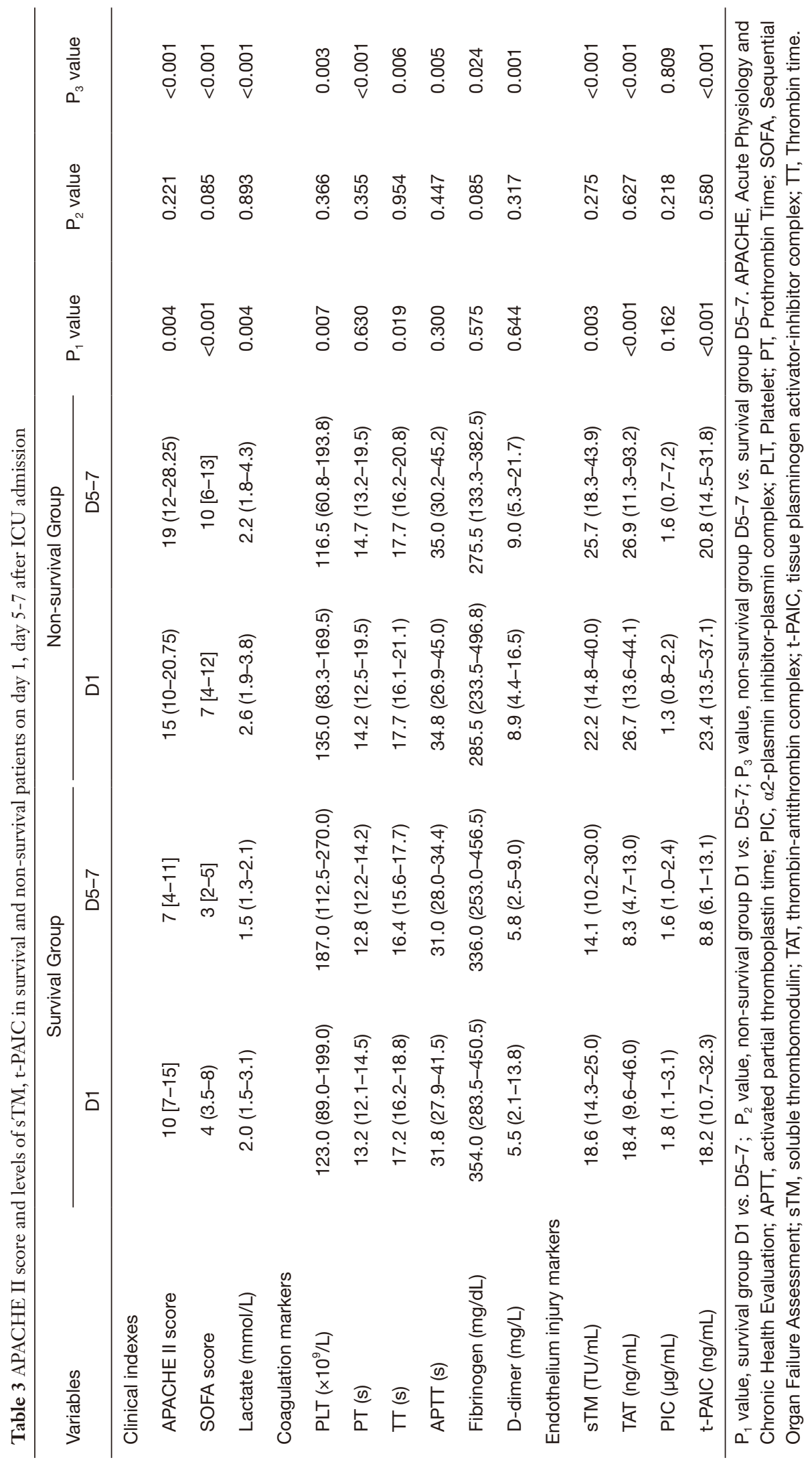


Table 4 Baseline characteristic and univariate analysis of the endothelial cell injury and coagulation markers between shock and non-shock groups

\begin{tabular}{|c|c|c|c|}
\hline Variables & Shock & Non-shock & $P$ value \\
\hline Male, n (\%) & $25(53.2)$ & $70(58.3)$ & 0.420 \\
\hline Age (years) & $63[44-72]$ & $60[47-72]$ & 0.750 \\
\hline \multicolumn{4}{|l|}{ Complications, n (\%) } \\
\hline Chronic liver disease & $2(4.2)$ & $3(2.5)$ & 0.620 \\
\hline Chronic renal failure & 7 (14.9) & $10(8.3)$ & 0.260 \\
\hline Congestive heart failure & $6(12.8)$ & $13(10.8)$ & 0.790 \\
\hline Chronic pulmonary disease & $8(17.0)$ & $7(5.8)$ & 0.034 \\
\hline \multicolumn{4}{|l|}{ Outcomes } \\
\hline Hospital stay (d) & $17[6-24]$ & $20[7-28]$ & 0.210 \\
\hline Mortality, n (\%) & $30(63.8)$ & $49(38.3)$ & 0.010 \\
\hline \multicolumn{4}{|l|}{ Clinical indexes } \\
\hline APACHE II score & $18[11-28]$ & $10(7-15.75)$ & $<0.001$ \\
\hline SOFA score & $11[7-14]$ & $4[3-7]$ & $<0.001$ \\
\hline Lactate (mmol/L) & $3.3(1.8-4.8)$ & $2.1(1.4-2.7)$ & $<0.001$ \\
\hline \multicolumn{4}{|l|}{ Coagulation markers } \\
\hline $\operatorname{PLT}\left(\times 10^{9} / \mathrm{L}\right)$ & $76.0(38.0-132.0)$ & $163.0(107.3-224.5)$ & $<0.001$ \\
\hline \multicolumn{4}{|l|}{ Endothelium injury markers } \\
\hline sTM (TU/mL) & $32.3(20.1-48.6)$ & $18.6(13.7-28.0)$ & $<0.001$ \\
\hline TAT (ng/mL) & $21.3(14.2-59.8)$ & $20.0(9.6-46.2)$ & 0.204 \\
\hline $\mathrm{PIC}(\mu \mathrm{g} / \mathrm{mL})$ & $1.4(0.7-3.4)$ & $1.50(1.0-2.4)$ & 0.835 \\
\hline $\mathrm{t}$-PAIC (ng/mL) & $23.6(12.7-38.0)$ & $15.7(8.7-40.0)$ & 0.009 \\
\hline \multicolumn{4}{|l|}{ Sites of infection, $\mathrm{n}(\%)$} \\
\hline Lung & $31(65.9)$ & $82(68.3)$ & 0.710 \\
\hline Abdomen & $9(19.1)$ & $23(19.2)$ & 1.000 \\
\hline Bloodstream & $2(4.2)$ & $4(3.3)$ & 1.000 \\
\hline Urinary tract infection & $2(4.2)$ & $6(5.0)$ & 1.000 \\
\hline Others & $3(6.4)$ & $5(4.2)$ & 0.690 \\
\hline
\end{tabular}

Table 4 (continued) 
Table 4 (continued)

\begin{tabular}{lccc}
\hline Variables & Shock & Non-shock & P value \\
\hline Microbiology positive, n (\%) & $18(38.3)$ & $48(40.0)$ & 0.860 \\
Gram-positive & $6(33.3)$ & $19(39.6)$ & 0.780 \\
Gram-negative & $8(44.4)$ & $22(45.8)$ & 1.000 \\
Fungi & $2(11.1)$ & $1(2.1)$ & 0.180 \\
Mixed & $2(11.1)$ & $6(12.5)$ & 1.000 \\
Microbiology unknown & $29(61.7)$ & $72(60.0)$ & 0.860 \\
\hline
\end{tabular}

APACHE, Acute Physiology and Chronic Health Evaluation; APTT, activated partial thromboplastin time; PIC, $\alpha 2$-plasmin inhibitor-plasmin complex; PLT, Platelet; PT, Prothrombin Time; SOFA, Sequential Organ Failure Assessment; sTM, soluble thrombomodulin; TAT, thrombinantithrombin complex; t-PAIC, tissue plasminogen activator-inhibitor complex, TT Thrombin time.

Table 5 Baseline characteristic and univariate analysis of the endothelial cell injury and coagulation markers between DIC and non-DIC groups

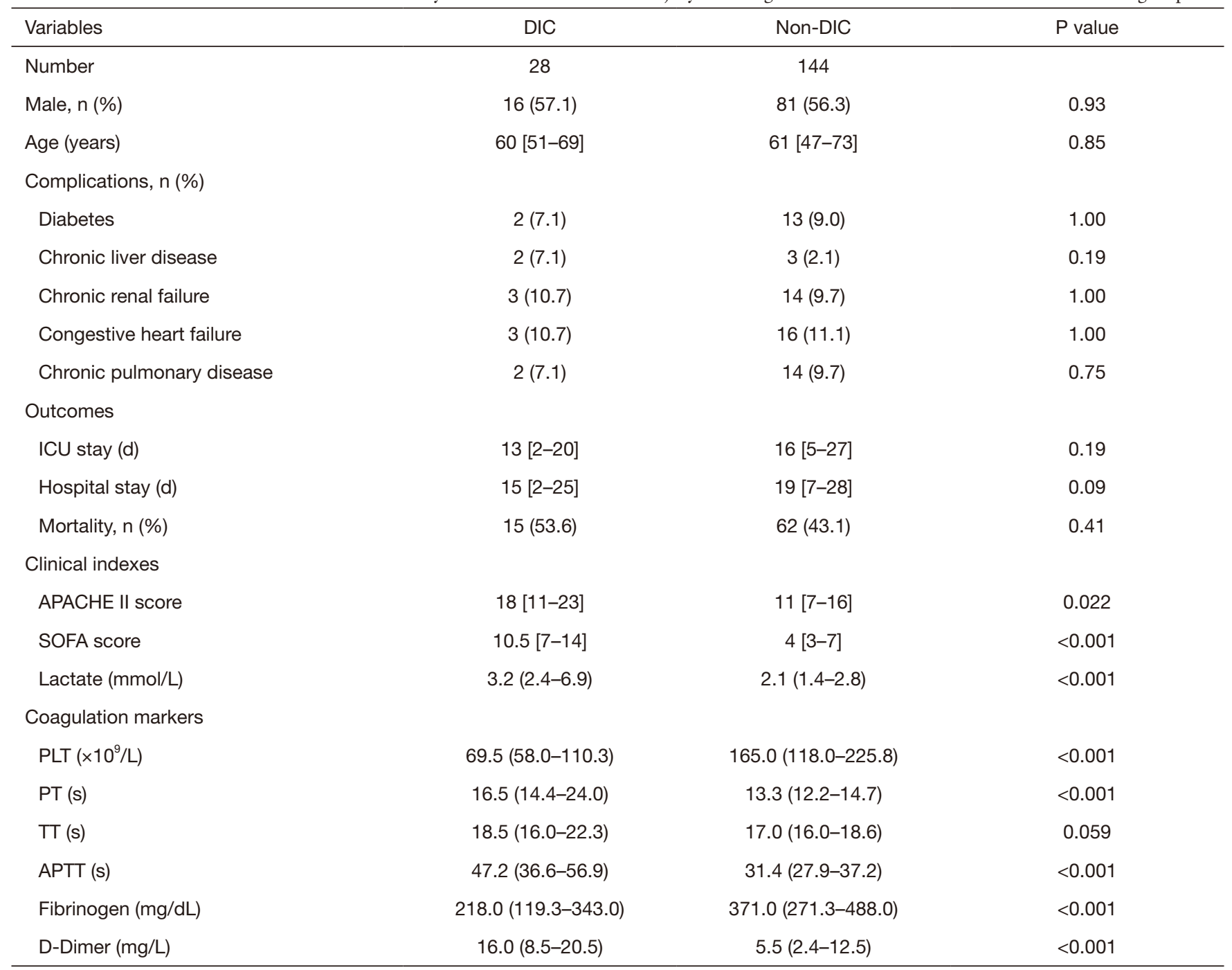

Table 5 (continued) 
Table 5 (continued)

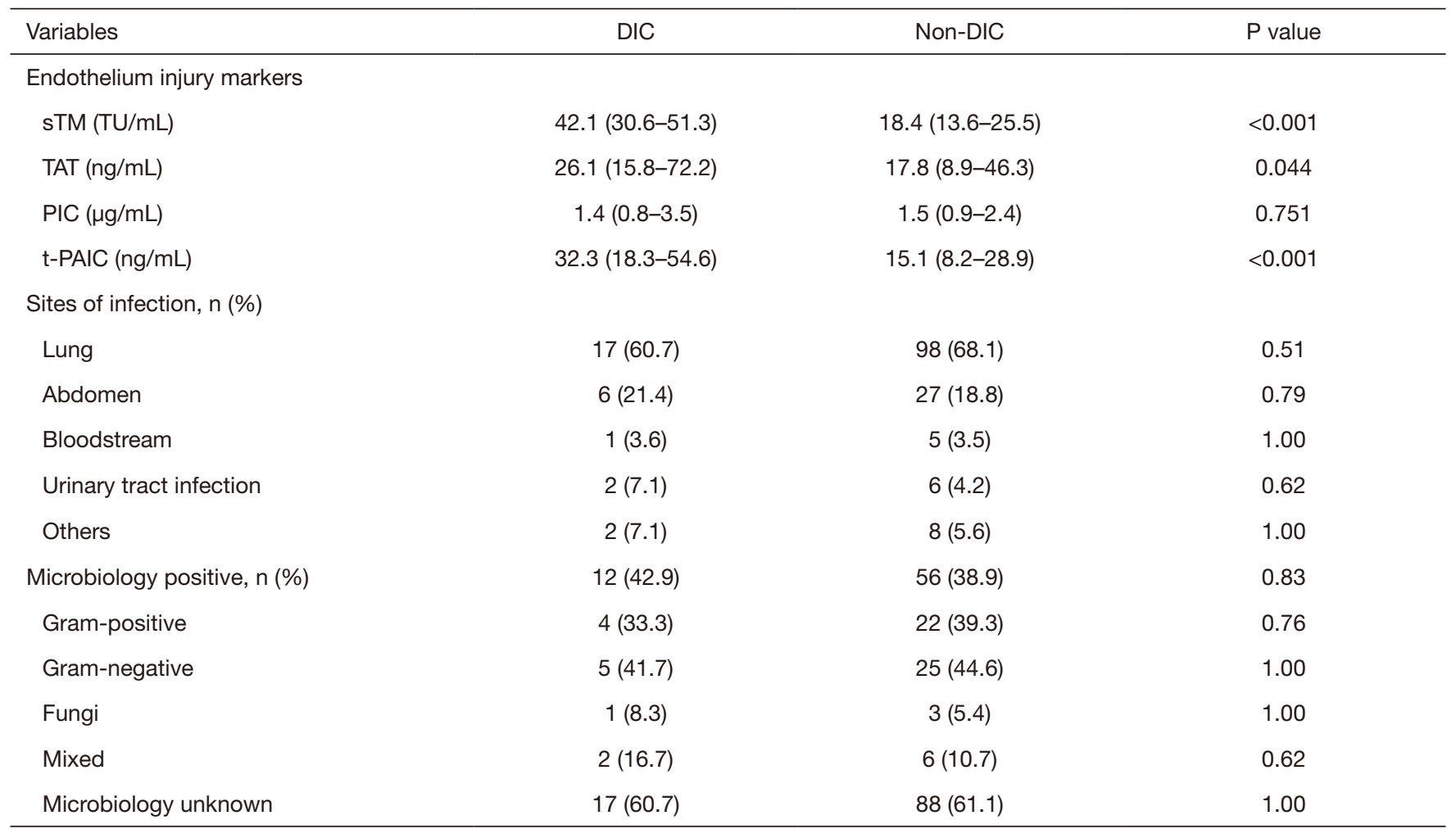

APACHE, Acute Physiology and Chronic Health Evaluation; APTT, activated partial thromboplastin time; PIC, $\alpha 2$-plasmin inhibitor-plasmin complex; PLT, platelet; PT, prothrombin time; SOFA, Sequential Organ Failure Assessment; sTM, soluble thrombomodulin; TAT, thrombinantithrombin complex; t-PAIC, tissue plasminogen activator-inhibitor complex; $\mathrm{TT}$, thrombin time.

plasminogen activator inhibitor-1 (PAI-1), and generate procoagulant microparticles (31-34). Endothelial cells are at the interface between inflammation and coagulation in sepsis (30). The excessive inflammatory response during sepsis leads to endothelial cell injury, which starts coagulation and causes the formation of microthrombi (38). At the same time, vascular endothelial cell injury causes peripheral vascular tension abnormalities, which leads to microcirculatory disorder. Our results showed that sTM and t-PAIC were independent factors for septic shock and DIC suggesting that endothelial injury which occurs as a result of inflammation initiation could in turn result in dysfunction of coagulation and even DIC. The prognostic value of endothelial markers was also studied by many studies (21), And this has been confirmed by our study that higher levels of sTM and t-PAIC are associated with increased 60-day mortality of sepsis.

Thrombomodulin (TM), a transmembrane glycoprotein, is abundant on all endothelial surfaces (39). As an essential component of the protein C system, endothelial-bound TM plays important roles in anti-coagulation (40). Soluble TM is the product of proteolytic degradation of endothelialbound TM. Under physiological conditions, sTM levels are in a stable range (41). When the endothelium is damaged, endothelial-bound TM is released from the endothelial surface, resulting in a significant increase in sTM concentration (42). As a sensitive marker of endothelial damage, sTM was found to be elevated in sepsis, DIC, vasculitis, venous thrombosis, trauma and ARDS patients (43). t-PAIC is a complex of t-PA and PAI-1. t-PA and PAI- 1 are mainly synthesized by endothelial cells. PAI1 can occur in various molecular forms in blood, including PAI-1 complexed with its target proteases (urokinasetype plasminogen activator and tissue-type plasminogen activator, t-PAIC). The increased concentration of t-PAIC suggests the occurrence of fibrin thrombosis and the sustained damage of endothelial cells, which can be used as a marker to determine the repair degree of the vascular endothelial system.

Increasing evidence has indicated that higher sTM and 
t-PAIC levels are indicative of endothelial injury, which showed potential values for the management of relevant diseases. A multicenter, prospective, observational study conducted by Mei et al. found that elevated plasma levels of sTM, t-PAIC and TAT were useful for the diagnosis of DIC (20). Another multicenter trial suggested that higher plasma sTM levels are associated with increased mortality in ARDS patients (22). Furthermore, Lin et al. found that increased serum TM levels are significantly correlated with illness severity and mortality in pediatric sepsis syndromes (44). Elevated plasma sTM levels are also associated with organ dysfunction in children with ARDS (23). However, only a few studies have focused on the relationship between the levels of sTM, t-PAIC, TAT and septic shock, sepsis-induced DIC. Our study indicated that sTM and t-PAIC are associated with a poor outcome of sepsis, and sTM was useful for the early prediction of DIC. The early prediction of septic shock is also crucial. Although many markers such as lactate level have been introduced for prediction of septic shock, those markers showed poor values in early prediction $(45,46)$. We found the increase of sTM can predict septic shock in early stage, which provides a solution to this issue.

TAT is also a sensitive marker of thrombin generation and development of DIC. But we did not confirm the association between increased level of TAT and sepsisinduced DIC. TAT level in patients without sepsis-induced has also increased due to coagulation dysfunction since they were also diagnosed with sepsis, and this narrowed the difference between two groups. Also, platelets might not be totally discarded after one-time centrifugation of blood samples, this could influence the preciseness of testing results of TAT.

The highlight of our study is that we explored the predictive value of endothelial markers for septic shock and sepsis-induced DIC, which added to the results of previous studies. This study provides two references for the clinicians. (I) A clinician should be aware of the development of sepsis if a patient shows elevated levels of endothelial markers. (II) If a patient has already been diagnosed with sepsis, elevated levels of endothelial markers indicate the higher possibility of developing septic shock, sepsis-induced DIC and poor 60-day outcome, which calls for the appropriate treatment strategy by the clinicians.

Our study had several limitations. First, this study was not a randomized controlled trial, and multiple unmeasured variables might account for the outcome difference observed in the study. Second, power analysis indicated that the analyses for TAT and PIC did not have enough statistical power. Thus, further studies with larger sample size are needed. Third, the testing protocols are varied among different institutes and the results are needed to be verified. The improvement of testing techniques is also crucial to the preciseness of the results.

\section{Conclusions}

The results of our study provide evidence that most patients developed severe coagulopathy and endothelial cell injury in the initial phase of sepsis, which was demonstrated by abnormalities in endothelial biomarkers and their strong association with 60-day prognosis and the development of septic shock and sepsis-induced DIC. In particular, the endothelial cell injury markers such as sTM and t-PAIC allowed early prediction of severe coagulopathy and poor outcome, leading to early intervention for patients with sepsis.

\section{Acknowledgments}

We would like to express our gratitude to all participants for their contributions to the study.

Funding: This work was supported by Key Project of Shanghai Municipal Health Bureau (2016ZB0202).

\section{Footnote}

Reporting Checklist: The authors have completed the STROBE reporting checklist. Available at https://dx.doi. org/10.21037/apm-21-2222

Data Sharing Statement: Available at https://dx.doi. org/10.21037/apm-21-2222

Conflicts of Interest: All authors have completed the ICMJE uniform disclosure form (available at https://dx.doi. org/10.21037/apm-21-2222). The authors have no conflicts of interest to declare.

Ethical Statement: The authors are accountable for all aspects of the work in ensuring that questions related to the accuracy or integrity of any part of the work are appropriately investigated and resolved. The study was conducted in accordance with the Declaration of Helsinki (as revised in 2013). The study was approved by the Institutional Research Ethics Committee of Zhongshan 
Hospital, Fudan University (No: 2006-23). Informed consent for the use of biological samples were acquired from patients or their authorized agents.

Open Access Statement: This is an Open Access article distributed in accordance with the Creative Commons Attribution-NonCommercial-NoDerivs 4.0 International License (CC BY-NC-ND 4.0), which permits the noncommercial replication and distribution of the article with the strict proviso that no changes or edits are made and the original work is properly cited (including links to both the formal publication through the relevant DOI and the license). See: https://creativecommons.org/licenses/by-nc-nd/4.0/.

\section{References}

1. Singer M, Deutschman CS, Seymour CW, et al. The Third International Consensus Definitions for Sepsis and Septic Shock (Sepsis-3). JAMA 2016;315:801-10.

2. Chen XH, Yin YJ, Zhang JX. Sepsis and immune response. World J Emerg Med 2011;2:88-92.

3. Iwashyna TJ, Cooke CR, Wunsch H, et al. Population burden of long-term survivorship after severe sepsis in older Americans. J Am Geriatr Soc 2012;60:1070-7.

4. Gaieski DF, Edwards JM, Kallan MJ, et al. Benchmarking the incidence and mortality of severe sepsis in the United States. Crit Care Med 2013;41:1167-74.

5. Dellinger RP, Levy MM, Rhodes A, et al. Surviving Sepsis Campaign: international guidelines for management of severe sepsis and septic shock, 2012. Intensive Care Med 2013;39:165-228.

6. Rhee C, Gohil S, Klompas M. Regulatory mandates for sepsis care--reasons for caution. N Engl J Med 2014;370:1673-6.

7. Vincent JL, Marshall JC, Namendys-Silva SA, et al. Assessment of the worldwide burden of critical illness: the intensive care over nations (ICON) audit. Lancet Respir Med 2014;2:380-6.

8. Fleischmann C, Scherag A, Adhikari NK, et al. Assessment of Global Incidence and Mortality of Hospital-treated Sepsis. Current Estimates and Limitations. Am J Respir Crit Care Med 2016;193:259-72.

9. Gando S, Levi M, Toh CH. Disseminated intravascular coagulation. Nat Rev Dis Primers 2016;2:16037.

10. Levi M. The coagulant response in sepsis. Clin Chest Med 2008;29:627-42, viii.

11. Hunt BJ. Bleeding and coagulopathies in critical care. N Engl J Med 2014;370:847-59.
12. Levi M, de Jonge E, van der Poll T, et al. Disseminated intravascular coagulation. Thromb Haemost 1999;82:695-705.

13. Shapiro NI, Schuetz P, Yano K, et al. The association of endothelial cell signaling, severity of illness, and organ dysfunction in sepsis. Crit Care 2010;14:R182.

14. Skibsted S, Jones AE, Puskarich MA, et al. Biomarkers of endothelial cell activation in early sepsis. Shock 2013;39:427-32.

15. Aird WC. The role of the endothelium in severe sepsis and multiple organ dysfunction syndrome. Blood 2003;101:3765-77.

16. Evans CE, Zhao YY. Impact of thrombosis on pulmonary endothelial injury and repair following sepsis. Am J Physiol Lung Cell Mol Physiol 2017;312:L441-51.

17. ProCESS Investigators; Yealy DM, Kellum JA, et al. A randomized trial of protocol-based care for early septic shock. N Engl J Med 2014;370:1683-93.

18. Hou PC, Filbin MR, Wang H, et al. Endothelial Permeability and Hemostasis in Septic Shock: Results From the ProCESS Trial. Chest 2017;152:22-31.

19. Kellum JA, Pike F, Yealy DM, et al. Relationship Between Alternative Resuscitation Strategies, Host Response and Injury Biomarkers, and Outcome in Septic Shock: Analysis of the Protocol-Based Care for Early Septic Shock Study. Crit Care Med 2017;45:438-45.

20. Mei H, Jiang Y, Luo L, et al. Evaluation the combined diagnostic value of TAT, PIC, tPAIC, and sTM in disseminated intravascular coagulation: A multi-center prospective observational study. Thromb Res 2019;173:20-6.

21. Yin Q, Liu B, Chen Y, et al. The role of soluble thrombomodulin in the risk stratification and prognosis evaluation of septic patients in the emergency department. Thromb Res 2013;132:471-6.

22. Sapru A, Calfee CS, Liu KD, et al. Plasma soluble thrombomodulin levels are associated with mortality in the acute respiratory distress syndrome. Intensive Care Med 2015;41:470-8.

23. Seymour CW, Liu VX, Iwashyna TJ, et al. Assessment of Clinical Criteria for Sepsis: For the Third International Consensus Definitions for Sepsis and Septic Shock (Sepsis-3). JAMA 2016;315:762-74.

24. Gando S, Iba T, Eguchi Y, et al. A multicenter, prospective validation of disseminated intravascular coagulation diagnostic criteria for critically ill patients: comparing current criteria. Crit Care Med 2006;34:625-31.

25. Rhodes A, Evans LE, Alhazzani W, et al. Surviving Sepsis Campaign: International Guidelines for Management 
of Sepsis and Septic Shock: 2016. Intensive Care Med 2017;43:304-77.

26. Orwoll BE, Spicer AC, Zinter MS, et al. Elevated soluble thrombomodulin is associated with organ failure and mortality in children with acute respiratory distress syndrome (ARDS): a prospective observational cohort study. Crit Care 2015;19:435.

27. Schouten $M$, Wiersinga WJ, Levi M, et al. Inflammation, endothelium, and coagulation in sepsis. J Leukoc Biol 2008;83:536-45.

28. Koyama K, Madoiwa S, Nunomiya S, et al. Combination of thrombin-antithrombin complex, plasminogen activator inhibitor-1, and protein C activity for early identification of severe coagulopathy in initial phase of sepsis: a prospective observational study. Crit Care 2014;18:R13.

29. Bombeli T, Mueller M, Haeberli A. Anticoagulant properties of the vascular endothelium. Thromb Haemost 1997;77:408-23.

30. Bevilacqua MP, Pober JS, Majeau GR, et al. Recombinant tumor necrosis factor induces procoagulant activity in cultured human vascular endothelium: characterization and comparison with the actions of interleukin 1. Proc Natl Acad Sci U S A 1986;83:4533-7.

31. Combes V, Simon AC, Grau GE, et al. In vitro generation of endothelial microparticles and possible prothrombotic activity in patients with lupus anticoagulant. J Clin Invest 1999;104:93-102.

32. Moore KL, Andreoli SP, Esmon NL, et al. Endotoxin enhances tissue factor and suppresses thrombomodulin expression of human vascular endothelium in vitro. J Clin Invest 1987;79:124-30.

33. Schleef RR, Bevilacqua MP, Sawdey M, et al. Cytokine activation of vascular endothelium. Effects on tissue-type plasminogen activator and type 1 plasminogen activator inhibitor. J Biol Chem 1988;263:5797-803.

34. Koyama K, Madoiwa S, Nunomiya S, et al. Combination of thrombin-antithrombin complex, plasminogen activator inhibitor-1, and protein $\mathrm{C}$ activity for early identification of severe coagulopathy in initial phase of sepsis: a prospective observational study. Crit Care 2014;18:R13.

35. Weiler H, Lindner V, Kerlin B, et al. Characterization of a mouse model for thrombomodulin deficiency. Arterioscler Thromb Vasc Biol 2001;21:1531-7.

36. Mavrommatis AC, Theodoridis T, Economou M, et al. Activation of the fibrinolytic system and utilization of the coagulation inhibitors in sepsis: comparison with severe sepsis and septic shock. Intensive Care Med 2001;27:1853-9.

37. Faust SN, Heyderman RS, Levin M. Coagulation in severe sepsis: a central role for thrombomodulin and activated protein C. Crit Care Med 2001;29:S62-7; discussion S67-8.

38. Xue M, Sun Z, Shao M, et al. Diagnostic and prognostic utility of tissue factor for severe sepsis and sepsis-induced acute lung injury. J Transl Med 2015;13:172.

39. Conway EM. Thrombomodulin and its role in inflammation. Semin Immunopathol 2012;34:107-25.

40. Takano S, Kimura S, Ohdama S, et al. Plasma thrombomodulin in health and diseases. Blood 1990;76:2024-9.

41. Boehme MW, Deng Y, Raeth U, et al. Release of thrombomodulin from endothelial cells by concerted action of TNF-alpha and neutrophils: in vivo and in vitro studies. Immunology 1996;87:134-40.

42. Ikegami K, Suzuki Y, Yukioka T, et al. Endothelial cell injury, as quantified by the soluble thrombomodulin level, predicts sepsis/multiple organ dysfunction syndrome after blunt trauma. J Trauma 1998;44:789-94; discussion 794-5.

43. Brogren H, Sihlbom C, Wallmark K, et al. Heterogeneous glycosylation patterns of human PAI-1 may reveal its cellular origin. Thromb Res 2008;122:271-81.

44. Lin JJ, Hsiao HJ, Chan OW, et al. Increased serum thrombomodulin level is associated with disease severity and mortality in pediatric sepsis. PLoS One 2017;12:e0182324.

45. Mikkelsen ME, Miltiades AN, Gaieski DF, et al. Serum lactate is associated with mortality in severe sepsis independent of organ failure and shock. Crit Care Med 2009;37:1670-7.

46. Ding H, Cao XY, Ma XG, et al. Endothelial cell injury with inflammatory cytokine and coagulation in patients with sepsis. World J Emerg Med 2013;4:285-9.

Cite this article as: Zhang J, Xue M, Chen Y, Liu C, Kuang Z, Mu S, Wei W, Yin J, Xiang H, Hu Y, Long X, Fang S, Sun S, Wang B, Tong C, Song Z. Identification of soluble thrombomodulin and tissue plasminogen activator-inhibitor complex as biomarkers for prognosis and early evaluation of septic shock and sepsis-induced disseminated intravascular coagulation. Ann Palliat Med 2021;10(10):10170-10184. doi: 10.21037/apm-21-2222 